\title{
Numerical simulations of graphene conductivity with realistic inter-electron interaction potential
}

\author{
D.L. Boyda* \\ Far Eastern Federal University, Vladivostok, 690091 Russia \\ E-mail: boyda_demail.ru \\ V.V. Braguta \\ Institute for High Energy Physics, Protvino, 142281 Russia \\ Institute of Theoretical and Experimental Physics, Moscow, 117218 Russia \\ E-mail: braguta@mail.ru
}

\section{M.V. Ulybyshev}

Institute of Theoretical Physics, University of Regensburg, D-93053 Germany, Regensburg, Universitatsstrasse 31

Institute for Theoretical Problems of Microphysics, Moscow State University, Moscow, 119899

Russia E-mail: Maksim. Ulybyshev@physik.uni-regensburg.de

There was a long time disagreement on semimetal-insulator phase transition in graphene between theoretical calculations and experimental data. The first results allowing to resolve this issue were obtained in the papers [Phys. Rev. Lett. 111, 56801 (2013)] and [Phys. Rev. B 89, 195429 (2014)] where antiferromagnetic order parameter was studied taking into account screening of Coulomb potential by $\sigma$-electrons of carbon atoms. Authors found that the appearance of antiferromagnetic (AFM) order is shifted into unphysical region of interaction strength. The appearance of AFM order parameter should be accompanied by the rapid fall of conductivity. Therefore the calculations of graphene conductivity taking into account screening of Coulomb potential by $\sigma$-electrons should be performed in order to demonstrate explicitly the phase transition to insulating state. We performed Monte-Carlo simulations of graphene tight-biding model with realistic electron-electron interaction and calculated the dependence of conductivity on dielectric permittivity of substrate. According to our calculations suspended graphene has conductivity $\sigma=(0.325 \pm 0.003) e 2 / \hbar$ which is very close to experimental value $\sigma=(1.01 \pm 0.04) e^{2} / 4 \hbar$. The phase transition in conductivity is also shifted into unphysical region $\varepsilon<1$ due to influence of $\sigma$-electrons.

The 33rd International Symposium on Lattice Field Theory

14 - 18 July 2015

Kobe International Conference Center, Kobe, Japan*

${ }^{*}$ Speaker. 


\section{Introduction}

Graphene is two-dimensional material with hexagonal lattice [1]. At low energies electrons in graphene can be described by effective field theory of Dirac massless fermions with Fermi velocity $v_{F}=c / 300$ [2]. Due to smallness of Fermi velocity electron-electron interaction in graphene can be described by instantaneous Coulomb potential with good precision. When graphene sheet is located on a substrate with dielectric permittivity $\varepsilon$ the interaction is decreased in $\frac{2}{1+\varepsilon}$ times. Thereby the strength of Coulomb potential can be changed through varying $\varepsilon$. Consequently one of the most interesting questions about graphene is whether the interaction at some $\varepsilon$ is strong enough to cause the semimetal - insulator phase transition.

In papers $[3,4]$ the chiral condensate in graphene effective field theory was studied with Monte-Carlo calculations and it has been shown that semimetal - insulator phase transition takes place at critical epsilon $\varepsilon_{c} \approx 4$. This result was confirmed by subsequent paper [5], where firstprinciple Monte - Carlo calculations of graphene tight -binding model with lattice Coulomb potential were conducted. In this work graphene conductivity was also calculated: in region where $\varepsilon>\varepsilon_{c}$ conductivity acquires constant value $\sigma \simeq 0.32 e^{2} / \hbar$ and tends to zero when $\varepsilon<\varepsilon_{c}$. In contrast, experiments $[6,7,8]$ reported the absence of the phase transition. Even suspended graphene with the strongest interaction is a conductor with conductivity $\sigma=(1.01 \pm 0.04) e^{2} / 4 \hbar$.

This disagreement between theory and experiments had not been understood until the work [9] was published. Authors of this work performed direct Hybrid Monte-Carlo simulations of graphene tight-biding model taking into account screening of Coulomb potential by $\sigma$-electrons of carbon atoms. It was demonstrated that fermionic condensate (antiferromagnetic order parameter) appears only when dielectric permittivity $\varepsilon<0.7$ thus the phase transition is shifted into unphysical region. Unfortunately, calculation of conductivity was not carried out and question about theoretical value of suspended graphene conductivity still remains open. In order to close this gap we studied whether effects of screening by $\sigma$-electrons of carbon atoms can change graphene conductivity.

\section{Model}

Full Hamiltonian consists of two terms $\hat{H}=\hat{H}_{t b}+\hat{H}_{\text {int }}$. The first term is tight-binding Hamiltonian. It describes free electrons in graphene:

$$
\hat{H}_{t b}=-\kappa \sum_{\langle x, y\rangle}\left(\hat{a}_{x}^{\dagger} \hat{a}_{y}+\hat{b}_{x}^{\dagger} \hat{b}_{y}+\text { c.c. }\right) \pm \sum_{x} m\left(\hat{a}_{x}^{\dagger} \hat{a}_{y}+\hat{b}_{x}^{\dagger} \hat{b}_{y}\right) .
$$

where $\kappa=2.8 \mathrm{eV}$ is the hopping parameter, $\hat{a}_{x}^{\dagger}\left(\hat{a}_{x}\right)$ and $\hat{b}_{x}^{\dagger}\left(\hat{b}_{x}\right)$ - creation (annihilation) operator for electron and hole respectively. The sum $\sum_{\langle x, y\rangle}$ is taken over all pairs of nearest-neighbors $x$ and $y$. Also we introduced mass term to eliminate zero mode of fermionic operator (see below). The mass term is unphysical thus we conducted all calculations with small masses and performed extrapolation to zero mass.

The interaction Hamiltonian $\hat{H}_{\text {int }}$ is:

$$
\hat{H}_{i n t}=\frac{1}{2} \sum_{x, y} \hat{q}_{x} V_{x y} \hat{q}_{y},
$$


where $\hat{q}_{x}=\hat{a}_{x}^{\dagger} \hat{a}_{x}-\hat{b}_{x}^{\dagger} \hat{b}_{x}$ is charge operator and $V_{x y}$ are matrix elements of inter-electron interaction potential. As it has been shown in [9] the Coulomb potential is not sufficient to cover physics of graphene thus we used more complicated interaction matrix. At small distances it is important to take into account screening of Coulomb potential by $\sigma$-electrons. Corresponding matrix elements $V_{x y}$ were calculated in [10]. At the same time at large distance the effect of screening is negligible and ordinary Coulomb potential can be safely used for the calculation of $V_{x y}$. For more details one can refer to the paper [9].

Using analogy with the lattice QCD techniques $[11,12]$ we constructed the partition function $Z=\operatorname{Tr} e^{-\beta \hat{H}}(\beta=1 / T$ is inverse temperature) by integrating out fermionic fields. HubbardStratonovich transformation is used to get rid of four-fermionic terms in the interaction Hamiltonian [13]. After some algebra we obtained the partition function in the form appropriate for Monte-Carlo simulations:

$$
Z=\int \mathscr{D} \phi e^{-S\left[\phi_{x, t}\right]}\left|\operatorname{det} M\left[\phi_{x, t}\right]\right|^{2}
$$

where $S\left[\phi_{x, t}\right]=\sum_{t=0}^{N_{t}-1} \sum_{x y} \phi_{x, t} V_{x y} \phi_{y, t}$ describes effects of interaction and $M\left[\phi_{x, t}\right]$ is fermionic matrix:

$$
\begin{aligned}
& \sum_{x, y, t, t^{\prime}}\left(\psi_{x, t}^{*} M_{x, y, t, t^{\prime}} \psi_{y, t^{\prime}}\right)= \\
& \sum_{t=0}^{N_{t}-1}\left[\sum_{x} \psi_{x, 2 t}^{*}\left(\psi_{x, 2 t}-\psi_{x, 2 t+1}\right)-\delta \kappa \sum_{\langle x, y\rangle}\left(\psi_{x, 2 t}^{*} \psi_{y, 2 t+1}+\psi_{y, 2 t}^{*} \psi_{x, 2 t+1}\right)\right. \\
& \left.\quad+\sum_{x} \psi_{x, 2 t+1}^{*}\left(\psi_{x, 2 t+1}-e^{-i \delta \phi_{x, t}} \psi_{x, 2 t+2}\right) \pm \delta m \sum_{x} \psi_{x, 2 t}^{*} \psi_{x, 2 t+1}\right]
\end{aligned}
$$

The integrand can be interpreted as Boltzmann weight factor hence we can generate configurations of Hubbard field $\phi_{x, t}$ with this probability distribution and calculate physical observable as average over generated configurations. It worth to note that squared determinant is not coincidence. It appears due to particle-antiparticle symmetry in graphene. Thus we don't have any sign problem and can use Hybrid Monte-Carlo algorithm to generate Hubbard field configurations. Some details can be found in $[9,5,13]$.

Current-current correlator is defined in usual way. Its calculation is performed according to lattice QCD techniques $[11,12]$. The current-current correlator can be written in the following form in terms of matrix elements of fermionic operator:

$$
G(\tau)=-\sum_{b, c} \frac{2 \vec{e}_{b} \vec{e}_{c}}{3 \sqrt{3} a^{2} N_{x} N_{y}}\left\langle\operatorname{Re} \operatorname{Tr}\left(j_{b} M^{-1}(0, \tau) j_{c} M^{-1}(\tau, 0)()\right\rangle\right.
$$

Here $\vec{e}_{b}\left(\vec{e}_{c}\right)$ are unit vectors in link directions $b(c)$ of graphene hexagonal lattice, $a=0.142 \mathrm{~nm}$ is graphene lattice spacing, $N_{x}$ and $N_{y}$ are the lattice sizes and $j_{a}$ is the matrix of one-particle current operator. Here we wrote only the connected term. As it has been shown for Coulomb potential in [5] all disconnected contributions in current-current correlator are much smaller than connected ones. Their relative statistical errors are also much larger so one needs substantially larger amount of supercomputer time to calculate disconnected contributions with good precision. In our case they are even smaller due to smallness of interaction potential in comparison with Coulomb one. Therefore we postponed calculation of disconnected contributions to future work and studied only connected term of current-current correlator. 


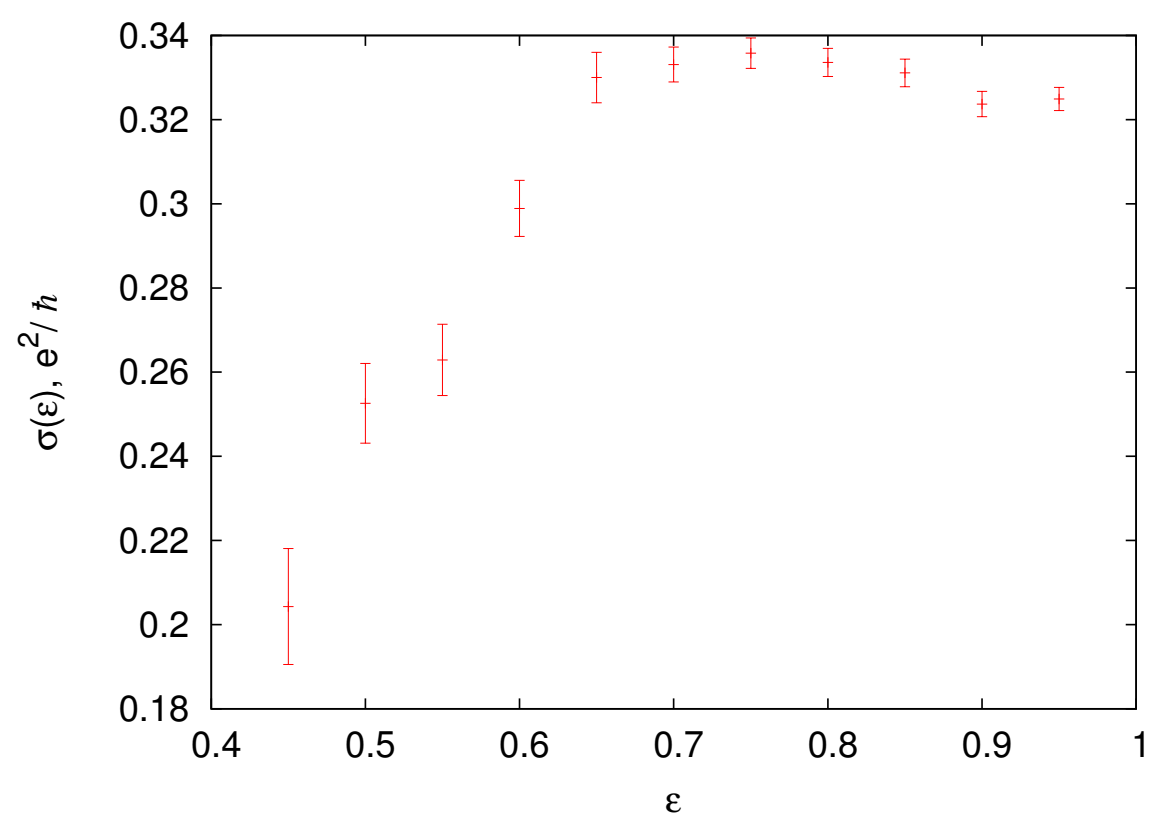

Figure 1: Dependence of graphene conductivity on dielectric permittivity of substrate. Calculation was done on lattice $18 \times 18 \times 20$ with $\mathrm{T}=0.5 \mathrm{eV}$ using 80 statistically independent configurations for each data point.

\section{Results and conclusions}

Conductivity can be extracted from current-current correlator with the help of Green-Kubo relation $[14,15]$. Commonly used method to invert Green-Kubo relation is maximum entropy method (MEM). Unfortunately it's hard to extract graphene conductivity using MEM in physically interesting region of temperatures even in the free theory (see [5]). So we followed [5] and calculated conductivity smeared over small frequencies:

$$
\bar{\sigma}=\mathscr{N}^{-1} \int_{0}^{\infty} \frac{d w}{2 \pi} \frac{2 w}{\sinh \left(\frac{w}{2 T}()\right.} \sigma\left(w()=\frac{1}{\pi T^{2}} G(\beta / 2() .\right.
$$

We performed calculations on lattices $N_{x}=N_{y}=18, N_{t}=20, \delta=0.1 \mathrm{eV}^{-1}$ with temperature $T=0.5 \mathrm{eV}$ and several values of $\varepsilon$. Several values of mass in the region $m=0.1-0.5 \mathrm{eV}$ were used. All data were extrapolated to zero mass using quadratic polynomial.

It is clearly seen from FIG. 1 that critical value $\varepsilon_{c} \approx 0.7$ separates semimetal and insulator phases: conductivity tends to constant value for $\varepsilon>\varepsilon_{c}$ and decreases when $\varepsilon<\varepsilon_{c}$. The calculated value of conductivity for suspended graphene $(\varepsilon=1)$ is $\sigma=0.325 \pm 0.003$ (in SI units $e 2 / \hbar$ ). Interestingly, it doesn't depend on the interaction strength above the critical value of $\varepsilon$. Calculated conductivity slightly overestimates its experimental value. We can explain it by the fact that we deal with conductivity smeared over small frequencies. Even in free theory it is lightly higher than the exact value of DC conductivity (see [5]).

Finally, we conclude that our calculations of conductivity are in full agreement with previous studies of AFM order parameter [9]. The screening of Coulomb potential at short distance shifts 
the transition in conductivity at the same critical $\varepsilon_{c} \approx 0.7$ which is in unphysical region. Our results confirm that below critical $\varepsilon$ graphene is in insulating phase. Very interestingly, the conductivity is quite stable in semimetal phase up to interaction strength which is very close to the critical one. This result confirms theoretical studies of conductivity renormalization in graphene where exact cancellation of all perturbative corrections was reported [16].

\section{Acknowledgements}

The work of MU was supported by the Alexander von Humboldt foundation (through the S. Kowalevskaja award to P. V. Buividovich) and by Grant RFBR-14-02-01261-a. The work of DB was supported by Far Eastern Federal University. The authors are grateful to FAIR-ITEP supercomputer center and to the supercomputer center of Moscow State University.

\section{References}

[1] Geim A. K., Novoselov K. S. // Nat. Mater. 2007. V. 6. P. 183.

[2] Castro Neto A. H., Guinea F., Peres N. M. R., Novoselov K. S., Geim A. K. // Rev. Mod. Phys. 2009. V. 81. P. 109.

[3] Drut J. E., Lahde T. A. //Phys. Rev. Lett. 2009. V. 102. P. 26802.

[4] Drut J. E., Lahde T. A. //Phys. Rev. Lett. 2009. V. 79. P. 165425.

[5] Buividovich P. V., Polikarpov M. I. //Phys. Rev. Lett. 2012. V. 86. P. 245117.

[6] Elias D. C., Gorbachev R. V., Mayorov A. S., Morozov S. V., Zhukov A. A., Blake P. , Ponomarenko L. A., Grigorieva I. V., Novoselov K. S., Guinea F. , Geim A. K. I/Nat. Phys. 2011. V. 7. P. 701.

[7] Mayorov A. S., Elias D. C., Mukhin I. S., Morozov S. V., Ponomarenko L. A., Novoselov K. S., Geim A. K., Grigorieva I. V. //Nano Lett. 2012. V. 12. P. 4629.

[8] Nair R. R., Blake P., Grigorenko A. N., Novoselov K. S., Booth T. J., Stauber T., Peres N. M. R., Geim A. K. //Science. 2008. V. 320. P. 1308.

[9] Ulybyshev M. V., Buividovich P. V., Katsnelson M. I., Polikarpov M. I. //Phys. Rev. Lett. 2013. V. 111. P. 56801.

[10] Wehling T. O., Şaşığ̆lu, Friedrich C., Lichtenstein A. I., Katsnelson M. I., Blügel S.. //Phys. Rev. Lett. 2011. V. 106. P. 236805.

[11] Gattringer C., Lang C. B. Quantum Chromodynamics on the Lattice: An Introductory Presentation. 2010. Berlin Heidelberg: Springer.

[12] Montvay I., Munster G. Quantum Fields on a Lattice. 1997. Cambridge: Cambridge University Press.

[13] Brower R. C. , Schaich D., Rebbi C. //PoS(Lattice 2011)056. 2011. California.

[14] Kadanoff Leo P., Martin Paul C. //Ann. Phys-New. York. 1963. V. 24, P. 419.

[15] Aarts G., Allton C., Foley J., Hands S., Kim S. //Phys. Rev. Lett. 2007. V. 99. P. 22002.

[16] A. Giuliani, V. Mastropietro, M. Porta //Phys. Rev. B 83, 195401 (2011). 